\title{
Current Saturation Due to Strong Field Concentration into Local Thin Area in Low-Density Polyethylene Sheet under Stepwise Increasing DC Voltage
}

\author{
Kazunori Kadowaki, ${ }^{*}$ Ryohei Ochi, and Ryotaro Ozaki \\ Department of Electrical and Electronic Engineering and Computer Science, Graduate School of Science \\ and Engineering, Ehime University, Bunkyo-cho 3, Matsuyama, Ehime 790-8577, Japan
}

(Received December 6, 2016; accepted June 9, 2017)

Keywords: current saturation, SCLC, field distortion, polyethylene, stepwise increasing voltage

Generally, space charge formation in dielectrics subjected to DC voltage results in the enhancement of a conduction current called space-charge-limited current (SCLC). In this study, we find an interesting phenomenon that the conduction current for a low-density polyethylene (LDPE) sheet does not increase with increasing applied voltage even though a large number of space charges are accumulated in its bulk. Simultaneous measurements of the conduction current and space charge distribution are carried out for a $150-\mu \mathrm{m}$-thick additive-free LDPE between a semiconduction anode and an aluminum-evaporated cathode. Under a low applied field of below $0.6 \mathrm{MV} / \mathrm{cm}$, the current is proportional to the square of the applied field because the maximum field strength in LDPE becomes higher than the mean applied field strength due to space charge formation in the LDPE bulk. Both the space charge distributions and the corresponding field distributions obtained by the simultaneous measurement roughly agree with the theoretical distributions based on the SCLC model. By increasing the applied field, however, the steady-state current saturates although a large number of positive space charges are accumulated in the LDPE bulk. We confirm that the steady-state current is restricted when a large number of frozen positive charges in front of the cathode are formed causing the strong field enhancement at a limited local thin area. These results imply that the carrier mobility at the local thin area was reduced by the excess field enhancement.

\section{Introduction}

Recently, DC power transmission technology has been introduced not only into conventional power transmission systems, but also into smart grid systems for the most efficient use of natural energy sources, such as offshore wind power plants and mega-solar plants. These applications for efficient power transmission will become widely used. One of the key parts for improving the long-term reliability of DC power systems is an insulation layer for DC cables. The breakdown of the insulation layer subjected to very high electrical stress for a long period will cause serious lifeline damage.

Generally, the insulation layer of the power cable is cross-linked by adding an organic peroxide into a low-density polyethylene (LDPE) in the manufacturing process because the insulation

*Corresponding author: e-mail: kadowaki.kazunori.mc@ehime-u.ac.jp

http://dx.doi.org/10.18494/SAM.2017.1541

ISSN 0914-4935 C MYU K.K. 
strength under a high temperature of over the melting point of LDPE is strongly improved by the cross-linking treatment. From the practical viewpoint, the insulation characteristics of the power cable should be evaluated for a test sample made with the cross-linked polyethylene (XLPE). However, the conduction mechanism of XLPE is more complex than that of LDPE because XLPE has many impurities produced by the cross-linking process. It is better to use LDPE rather than XLPE as the test sample in the first step to clarify the mechanisms of high field conduction and space charge dynamics in simple solid dielectrics. Therefore, there are many studies on space charge dynamics in LDPE under DC high fields. It is known that the space charge formation is associated with insulation degradation. ${ }^{(1-5)}$ In the last two decades, Auge et al. ${ }^{(6)}$ and Montanari et $a l^{(7)}$ have experimentally evaluated a critical applied field strength to start the electrical degradation by measuring not only space charge distribution, but also external current and electroluminescence (EL) under $1 \mathrm{MV} / \mathrm{cm}$. They confirmed that the critical applied field for space charge injection from a Au-evaporated electrode into the LDPE bulk was in good agreement with both the critical field for EL detection and that corresponding to the transition point of the conduction mechanism from the Ohmic model to the space-charge-limited current (SCLC) model.

It is known that the SCLC model is used to explain the current increasing phenomena due to space charge injection into the bulk of dielectrics. In most cases, the relationship between current density and applied field is explained by the Ohmic model until the applied field attains a critical level. With increasing applied field, the relationship cannot be explained by the Ohmic model but can be explained by the SCLC model. ${ }^{(8)}$ With further increasing applied voltage, an abrupt increase in current is often observed before breakdown occurs. However, the transition from the SCLC conduction model to the subsequent model associated with breakdown is still unclear. In particular, the relationship between the increasing current and the space charge dynamics in solid dielectrics under a high DC field over $1 \mathrm{MV} / \mathrm{cm}$ is not well understood. From this viewpoint, simultaneous measurements of current and space charge distribution in LDPE will give us valuable information. However, there are only several reports on the relationship between the steady-state current and the space charge dynamics in a wide range from the Ohmic conduction state to the final conduction state before breakdown. ${ }^{(9-12)}$

In this study, we measure simultaneously the current density and space charge distribution in a $150-\mu \mathrm{m}$-thick LDPE sheet subjected to stepwise increasing voltage. Experimental results show that the relationships between the current and the applied field are strongly affected by not only electrode materials but also an antioxidant additive in LDPE. ${ }^{(13)}$ In addition, an interesting phenomenon about the current-voltage characteristics is observed for an additive-free LDPE sheet between a semiconduction (SC) anode and an aluminum (Al)-evaporated cathode. Results show that the conduction current under a DC stepwise increasing voltage up to $30 \mathrm{kV}$ is saturated even though the space charge density in the LDPE bulk increases with increasing applied voltage. The reason for the current saturation will be discussed in this study.

\section{Materials and Methods}

\subsection{Samples and electrodes}

Additive-free polyethylene samples were prepared from a 150- $\mu \mathrm{m}$-thick LDPE sheet produced by high-temperature press forming (grade G201 by Sumitomo Chemical Industries). In 
experiments dealing with the effects of additives, samples were prepared from $100 \mathrm{~g}$ of LDPE kneaded with $5 \mathrm{~g}$ of phenol-based antioxidant (Irganox 1010, CIBA Japan).

An Al main electrode of $12 \mathrm{~mm}$ diameter was evaporated on the back of the LDPE sheet as a cathode. Around the main electrode, an Al-evaporated ring (inner diameter $20 \mathrm{~mm}$, outer diameter $28 \mathrm{~mm}$ ) was formed coaxially on the back of the sheet as a guard electrode to prevent the undesirable detection of the surface leakage current. On the upper side of the sample, either an Alevaporated electrode or a SC electrode made with a carbon-black-rich rubber was used as an anode of $9 \mathrm{~mm}$ diameter.

\subsection{Simultaneous measurement system}

Space charge distributions were measured simultaneously with external currents by the pulsedelectroacoustic (PEA) method with a picoammeter that we made. The experimental setup is shown in Fig. 1. A lower grounded central conductor made of aluminum was located on the central axis of the PEA measurement system and was surrounded coaxially by a grounded outer conductor. The central conductor acted not only as a current-sensing terminal but also as a pressure wave transmission rod. The bottom of the central conductor was alumited (anodized). The alumina $\left(\mathrm{Al}_{2} \mathrm{O}_{3}\right)$ layer acted as an insulator with a resistivity above $10^{14} \Omega$-cm. Since the thickness of the alumina layer was about $50 \mu \mathrm{m}$, undesirable current flow toward a piezoelectric sensor (KF PIEZOFILM, thickness: $9 \mu \mathrm{m}$, Kureha Chemical Industry Co.) was completely restricted, whereas pressure waves could propagate to the piezoelectric sensor almost without attenuation. The pressure wave signals were amplified by an amplifier (AU-1332, MITEQ), then observed with a digital oscilloscope (TDS520B, Tektronix). The current picked up by the central conductor was fed to the picoammeter, which consisted of a current-to-voltage conversion circuit with an operational amplifier (AD549JH, Analog Devices) and a subsequent operational amplifier (TLO81CP, Texas Instruments). The conversion ratio of the picoammeter was expressed as $V_{\text {out }}=2 \times 10^{8} I_{\text {in }}$, where

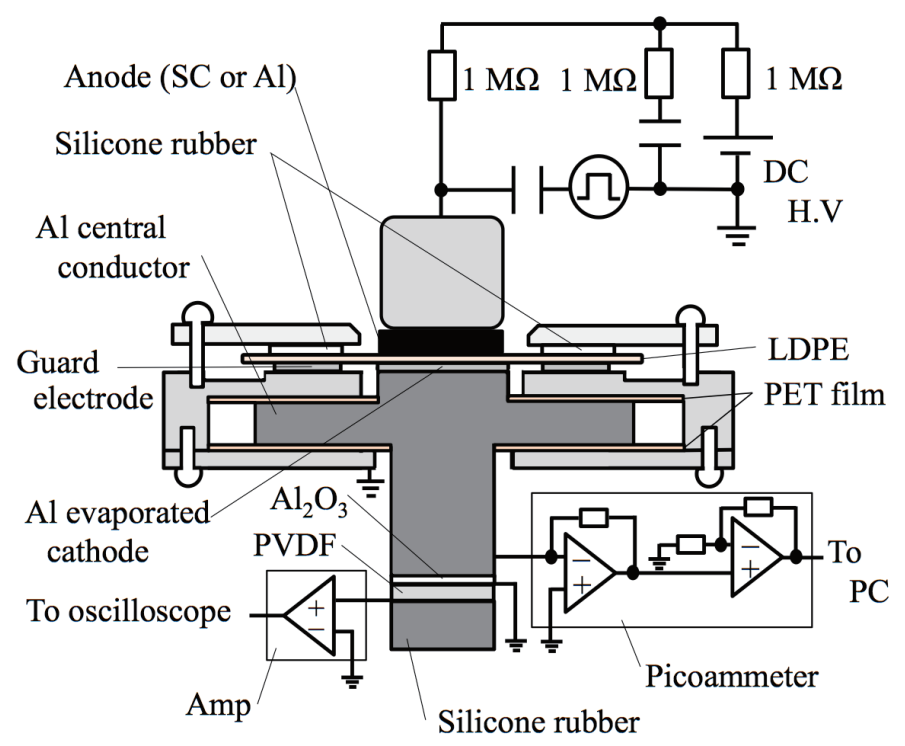

Fig. 1. (Color online) Sectional view of simultaneous measurement system for space charge distribution and external current. 
$V_{\text {out }}$ is output voltage and $I_{\text {in }}$ is input current. The effective measuring range of the picoammeter is on the order of $10^{-10}$ to $10^{-8} \mathrm{~A}$.

A pulse generator to produce pressure wave from space charges was operated at intervals of $10 \mathrm{~s}$, and the current waveform was recorded during this interval so as to prevent the effect of the pulsed voltage on the current measurement. That is, the space charge distribution and the external current were measured every $10 \mathrm{~s}$. A stepwise increasing DC voltage with $1.5 \mathrm{kV}$ per one step rise was applied to the sample for $1 \mathrm{~h}$ using a DC source (HARb-100R1.5, Matsusada Precision). To avoid breakdown caused by a rapid increase in voltage height, the applied voltage was gradually increased with a rise time of $10 \mathrm{~s}$ for each step. After the voltage increase was finished, the voltage height was fixed for $3 \mathrm{~min}$. This cycle was repeated 20 times, so that the voltage height was finally up to $30 \mathrm{kV}$, i.e., $2.0 \mathrm{MV} / \mathrm{cm}$ in mean field strength. All experiments were carried out at a temperature of $30 \pm 1{ }^{\circ} \mathrm{C}$.

\section{Results and Discussion}

\subsection{Effects of additional antioxidant and electrode material}

Temporal changes in the current during the stepwise increasing voltage were measured for the LDPE sheets with or without the antioxidant additive under $\mathrm{SC}(+) / \mathrm{Al}(-)$ or $\mathrm{Al}(+) / \mathrm{Al}(-)$ electrode systems. Typical current waveforms recorded for $1 \mathrm{~h}$ are shown in Fig. 2(a) for additive-free LDPE with $\mathrm{Al}(+) / \mathrm{Al}(-)$, in Fig. 2(b) for additive-containing LDPE with $\mathrm{SC}(+) / \mathrm{Al}(-)$, and in Fig. 2(c) for

(a)

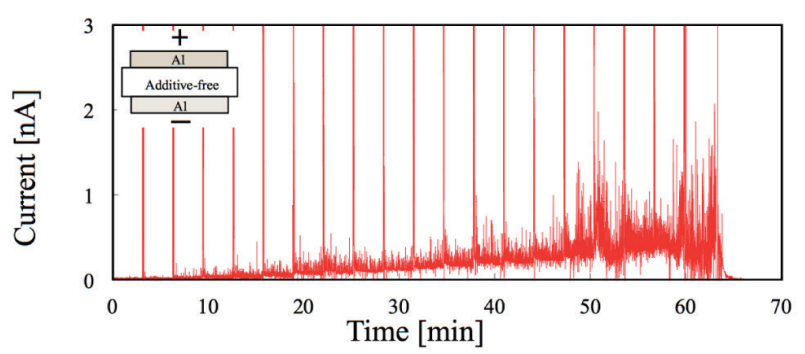

(b)

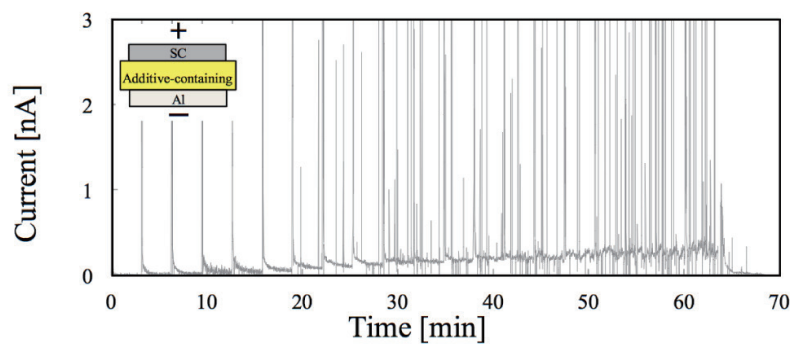

(c)

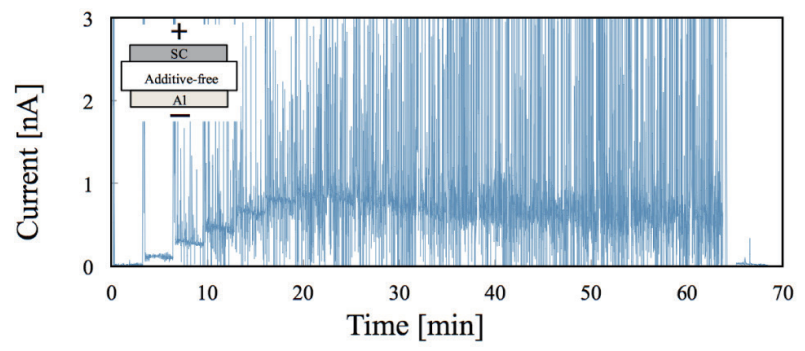

Fig. 2. (Color online) Temporal changes in external currents under stepwise increasing voltage for (a) additivefree LDPE with $\mathrm{Al}(+) / \mathrm{Al}(-)$, (b) additive-containing LDPE with $\mathrm{SC}(+) / \mathrm{Al}(-)$, and (c) additive-free LDPE with $\mathrm{SC}(+) / \mathrm{Al}(-)$. 
additive-free LDPE with $\mathrm{SC}(+) / \mathrm{Al}(-)$. The current of additive-free LDPE with $\mathrm{SC}(+) / \mathrm{Al}(-)$ in Fig. 2(c) is much higher than those of the other conditions. Conduction currents in solid dielectrics are limited not only by the trapping site in the bulk but also by a potential barrier at a dielectricelectrode contact. ${ }^{(14)}$ The reason why the conduction current for additive-free LDPE with $\mathrm{SC}(+) / \mathrm{Al}(-)$ is higher than the others is that many positive space charges from the $\mathrm{SC}$ anode can be drifted deeply into the bulk of LDPE without deep trapping sites. The barrier height at the SCLDPE contact must be much smaller than that of the Al-LDPE contact because there are many impurity levels between the valence band and the conduction band of the SC rubber, so that many positive charges can be injected from the SC electrode into the LDPE bulk. Therefore, the type of the conduction of LDPE with $\mathrm{SC}(+) / \mathrm{Al}(-)$ is classified as bulk-limited type. However, it is clearly observed that the conduction current of additive-containing LDPE in Fig. 2(b) is much lower than that of additive-free LDPE in Fig. 2(c), although these LDPE sheets are in contact with the SC electrode. This is because the phenolic antioxidant agent in LDPE acts as deep trapping sites for space charges. The injected space charges from the SC anode into additive-containing LDPE are trapped by the antioxidants and then they are frozen in the vicinity of the anode. In such a case, the current-voltage characteristic obeys the SCLC model as shown later. On the other hand, the current-voltage characteristic for the additive-free LDPE with the $\mathrm{Al}(+) / \mathrm{Al}(-)$ electrode under a low applied field cannot be explained by the SCLC model. This is because the charge injection is strongly restricted by a high potential barrier at the Al-LDPE contact, so called electrode-limited type. Owing to the high potential barrier at the contact, the current-voltage characteristic under the low applied field obeys the Ohmic model. When the applied field exceeds a critical level, the charges are eventually injected from the Al anode because the height in the potential barrier is reduced by the Schottky effect. In this situation, the conduction current is not limited by the electrode but limited by the LDPE bulk. Therefore, the conduction model is shifted from the Ohmic model to the SCLC model as the applied voltage increases.

We calculated the mean current density in each step from current data. As shown in Figs. 2(a)-2(c), repetitive large current pulses were observed at the interval of $3 \mathrm{~min}$. These large pulses were not due to the conduction current component produced by the very fast space charges moving in the LDPE bulk but due to the charging current (displacement current) component produced by the stepwise increase in the applied voltage. To eliminate the charging current components from the mean current, only current data measured in the last $1 \mathrm{~min}$ for each 3-min measurement was used for the calculation of the mean current. Relationships between the mean current density $J$ and the applied field $E=V / d$, where $V$ is applied voltage and $d$ is sheet thickness, are shown in Fig. 3 as double logarithmic plots. The slope of the dotted straight line in the double logarithmic plot means that the current density is proportional to $E$, whereas the slope of solid straight lines means that the current density is proportional to $E^{2}$. As shown in Fig. 3, the current density for additive-free LDPE with $\mathrm{Al}(+) / \mathrm{Al}(-)$ subjected to the applied field below $0.6 \mathrm{MV} / \mathrm{cm}$ is roughly proportional to $E$. This fact indicates that the $J-E$ characteristic obeys Ohm's law because space charges are scarcely injected from the Al electrodes under the low applied field so that the electric field distribution in the LDPE bulk must be uniform. The reason why the $J_{-}$ $E$ characteristic for additive-free LDPE with $\mathrm{Al}(+) / \mathrm{Al}(-)$ has a flexion point at $0.6 \mathrm{MV} / \mathrm{cm}$ is that a Schottky injection from the Al electrode into the LDPE bulk is caused by the higher applied field of over $0.6 \mathrm{MV} / \mathrm{cm}$ as shown later. The electric field distribution is distorted by these space charges accumulated in the LDPE bulk, so that the maximum field strength in the LDPE bulk becomes higher than $E$. The important point is that the drift velocity of carriers must be enhanced 


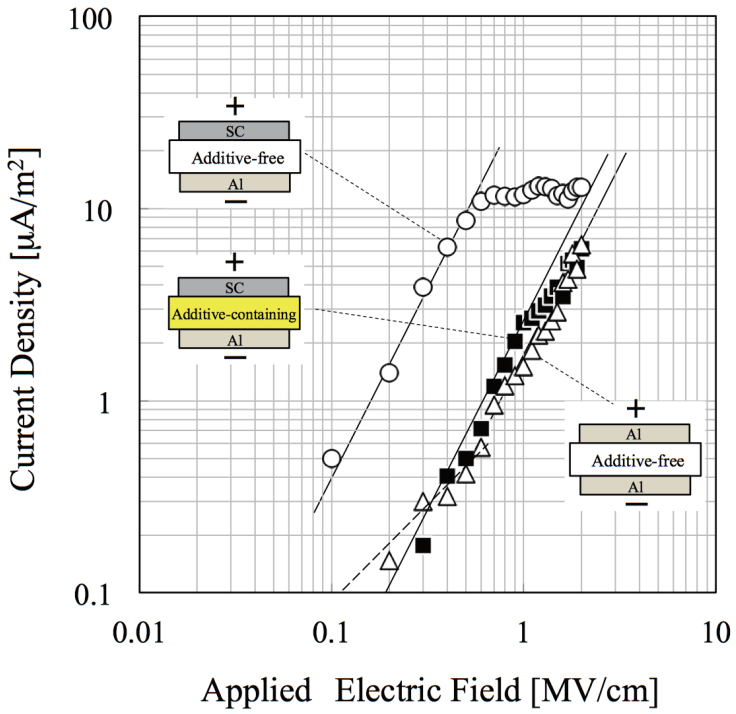

Fig. 3. (Color online) $J-E$ characteristics of the LDPE samples subjected to stepwise increasing voltage.

by the field concentration due to the injection of positive charges and subsequent accumulation. According to the SCLC model, the current density $J$ is expressed as ${ }^{(15,16)}$

$$
J=\frac{9}{8} \varepsilon \mu \frac{V^{2}}{d^{3}}=\frac{9}{8} \varepsilon \mu \frac{E^{2}}{d}
$$

where $\varepsilon$ is permittivity and $\mu$ is mobility. As expressed in Eq. (1), $J$ is proportional to $E^{2}$. Therefore, the $J-E$ characteristic for additive-free LDPE with the $\mathrm{Al}(+) / \mathrm{Al}(-)$ electrode indicates that the Ohmic conduction model at the initial stage of the stepwise voltage application is followed by the SCLC conduction model. On the other hand, the $J-E$ characteristics for the LDPE samples with $\mathrm{SC}(+) / \mathrm{Al}(-)$ electrodes do not obey Ohm's law even if the applied field is very low. This is because positive space charges can be easily injected from the SC anode as mentioned earlier. The $J-E$ characteristic for additive-containing LDPE with $\mathrm{SC}(+) / \mathrm{Al}(-)$ is proportional to $E^{2}$. The plots of $J$ measured at low $E$ of below $1 \mathrm{MV} / \mathrm{cm}$ are well fitted on the solid line. However, the plots are gradually away from the solid line with increasing $E$. The reason for the difference between the plots and the solid line at the higher field will be discussed later.

In the results of the $J-E$ characteristics shown in Fig. 3, the most remarkable point is the fact that the current density for additive-free LDPE with $\mathrm{SC}(+) / \mathrm{Al}(-)$ tends to saturate as $E$ increases. In the range of $E<0.6 \mathrm{MV} / \mathrm{cm}, J$ is proportional to $E^{2}$. However, in the range of $E>0.6 \mathrm{MV} / \mathrm{cm}, J$ is kept constant. It appears that the conductivity of LDPE decreases with increasing $E$.

Space charge distributions measured simultaneously with the currents and corresponding field distributions calculated by Poisson's equation are shown in Figs. 4(a) and 4(b), respectively, for additive-free LDPE with $\mathrm{Al}(+) / \mathrm{Al}(-)$. Space charges injected into the LDPE bulk are scarcely observed for additive-free LDPE with $\mathrm{Al}(+) / \mathrm{Al}(-)$ under low applied fields of below $0.6 \mathrm{MV} / \mathrm{cm}$, whereas they are observed at the applied fields higher than $0.6 \mathrm{MV} / \mathrm{cm}$. Therefore, field distortion due to space charge injection is also scarcely observed at the lower fields. The critical field strength for space charge injection agrees with that for the transition from the Ohmic model to the SCLC model. 

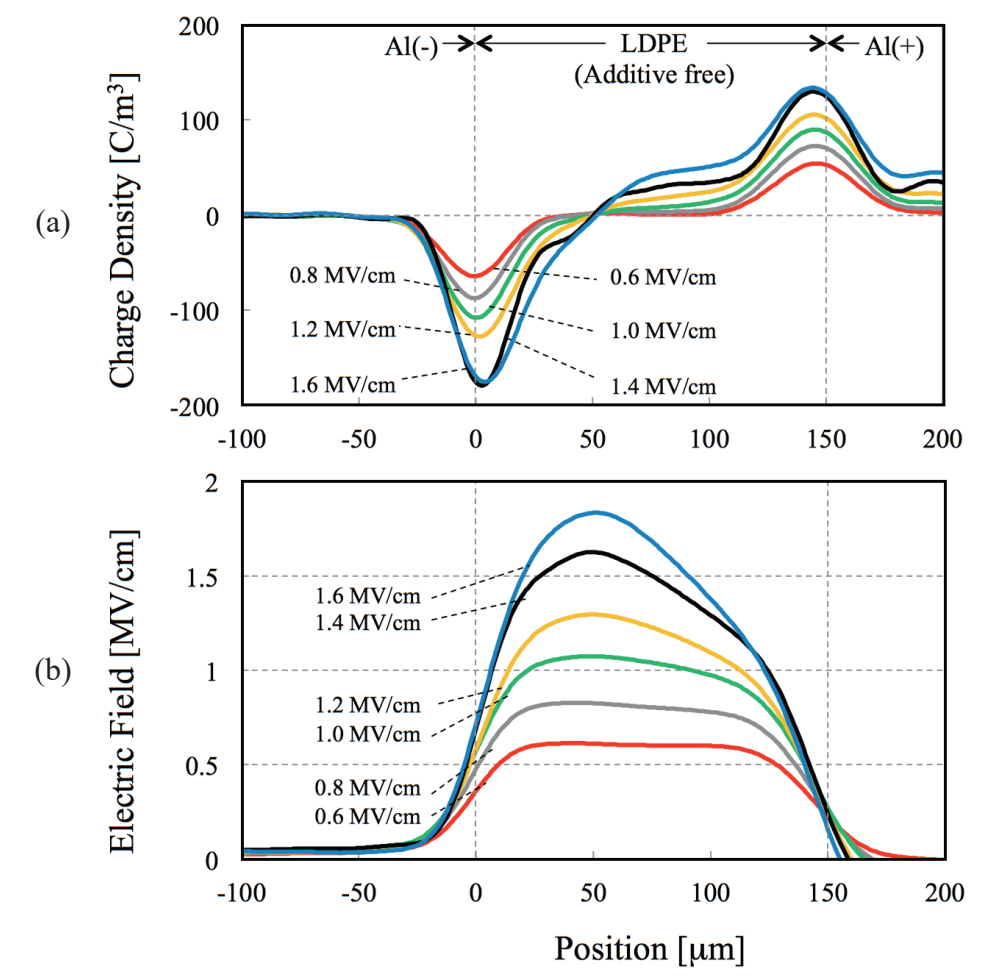

Fig. 4. (Color online) (a) Space charge distributions and (b) corresponding electric field distributions under various applied fields for additive-free LDPE with $\mathrm{Al}(+) / \mathrm{Al}(-)$.

Space charge distributions and corresponding field distributions are shown in Figs. 5(a) and 5(b), respectively, for additive-containing LDPE with $\mathrm{SC}(+) / \mathrm{Al}(-)$. In this case, space charges injected from the SC anode into the LDPE bulk are clearly observed at the initial stage in the stepwisely increasing voltage application. These positive space charges are accumulated in the LDPE bulk near the anode side, so that the field strength near the anode side becomes lower than that near the cathode side, as shown in Fig. 5(b). According to the SCLC model, the density of positive space charges injected from the anode into the LDPE bulk in the steady state can be expressed as

$$
\rho(x)=\frac{3}{4}\left(\frac{V}{d^{2}}\right)\left(\frac{d-x}{d}\right)^{-\frac{1}{2}},
$$

where $x$ refers to a location in the LDPE bulk; $x=0$ corresponds to the position of the Al cathode surface, whereas $x=150$ corresponds to the position of the SC anode surface. Equation (2) indicates that the charge density $\rho(x)$ as a function of $x$ is inversely proportional to the square root of $d-x$, which corresponds to the penetration depth of the charges from the anode surface. Figure 6 shows a theoretical distribution of the positive charge density $\rho(x)$ calculated with Eq. (2). The calculated profile of the injected positive charges indicates that the most injected positive 
(a)

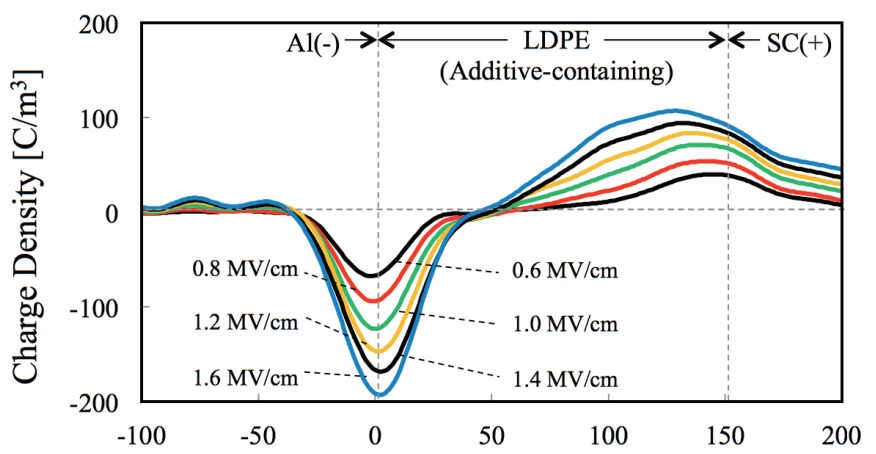

(b)

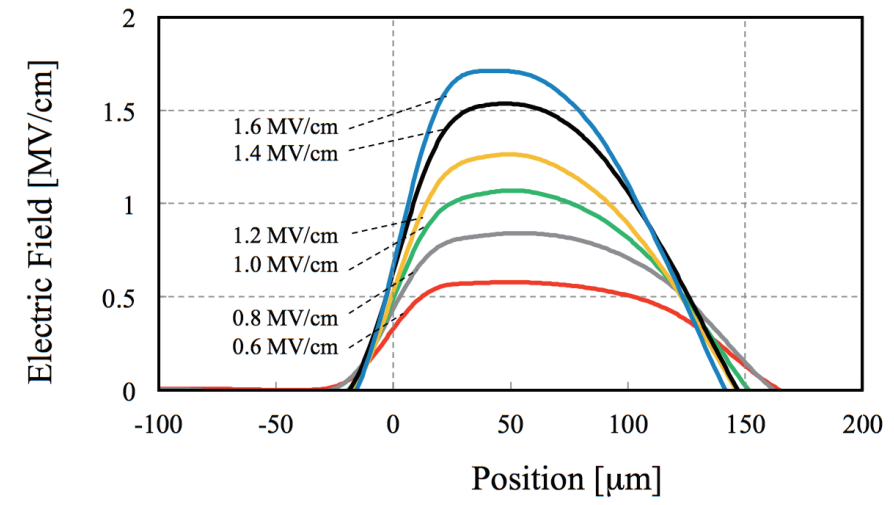

Fig. 5. (Color online) (a) Space charge distributions and (b) corresponding electric field distributions under various applied fields for additive-containing LDPE with $\mathrm{SC}(+) / \mathrm{Al}(-)$.

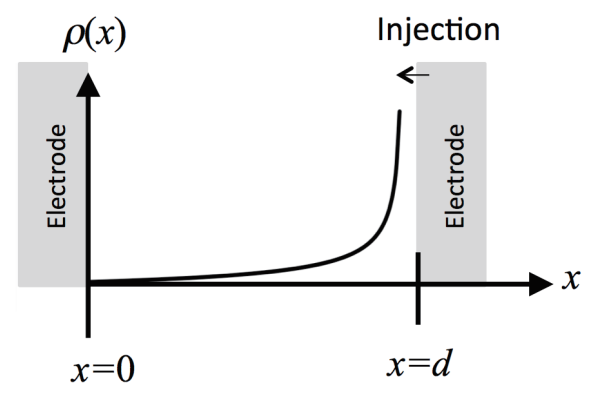

Fig. 6. A theoretical distribution of injected space charges from anode based on SCLC model.

charges are located in the vicinity of the anode. The charge density $\rho(x)$ decreases markedly with increasing distance from the anode surface. In comparison with the space charge distributions in Fig. 5(a), we can recognize that the experimental profiles of the positive charge distribution under the low applied field of below $0.8 \mathrm{MV} / \mathrm{cm}$ are fitted with the theoretical profile shown in Fig. 6. However, the experimental profiles under the higher applied field of over $1 \mathrm{MV} / \mathrm{cm}$ are less similar to the theoretical profiles in Fig. 6. The difference between the experimental profiles and the theoretical profile for additive-containing LDPE with $\mathrm{SC}(+) / \mathrm{Al}(-)$ must be closely related to the fact that the current density measured under the high applied field of over $1 \mathrm{MV} / \mathrm{cm}$ is not well fitted on the theoretical line based on the SCLC model, as shown in Fig. 3. 
Figures 7(a) and 7(b) show space charge distributions and corresponding field distributions for additive-free LDPE with $\mathrm{SC}(+) / \mathrm{Al}(-)$, respectively. Remarkable differences in the penetration depth of positive space charge are observed between Figs. 5(a) and 6(a). In the case of additivefree LDPE, the injected positive charges from the SC anode penetrate deeply into the LDPE bulk because there are no deep trapping sites produced by additives. Finally, the dense positive space charges are frozen in front of the Al cathode in the steady state although the field strength in the LDPE bulk near the cathode surface is strongly enhanced. ${ }^{(17,18)}$ When a constant field of over 1 $\mathrm{MV} / \mathrm{cm}$ was applied to the sample as a step function, the transport of the traveling positive charge packet from the SC anode into the LDPE bulk was clearly observed as reported by Matsui et $a l .{ }^{(17,18)}$ In the present study, however, the transport of the dense charge packet across the sample was not clearly observed. This was because the applied voltage was slowly increased step-by-step with the small step of $0.1 \mathrm{MV} / \mathrm{cm}$. As a result of the deep penetration of a large number of positive charges caused by the long time application of the stepwise increasing voltage, the maximum field strength $E_{\max }$ in LDPE becomes much higher than $E$. We define the field enhancement factor as $F E F=E_{\max } / E$. The results indicate that FEF increases with increasing applied field. For example, $F E F$ is up to 1.3 for $E=0.8 \mathrm{MV} / \mathrm{cm}, 1.4$ for $E=1.0 \mathrm{MV} / \mathrm{cm}, 1.7$ for $E=1.2 \mathrm{MV} / \mathrm{cm}, 1.8$ for $E=1.4$ $\mathrm{MV} / \mathrm{cm}$, and 1.9 for $E=1.6 \mathrm{MV} / \mathrm{cm}$. Owing to the positive charge accumulation in the LDPE bulk, the field strength increases with increasing distance from the anode surface. According to the SCLC model, $E(x)$ can be expressed as

(a)

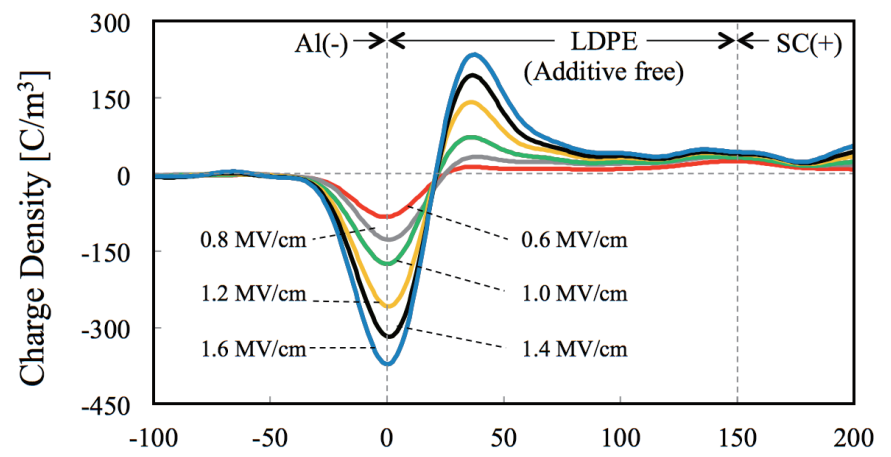

(b)

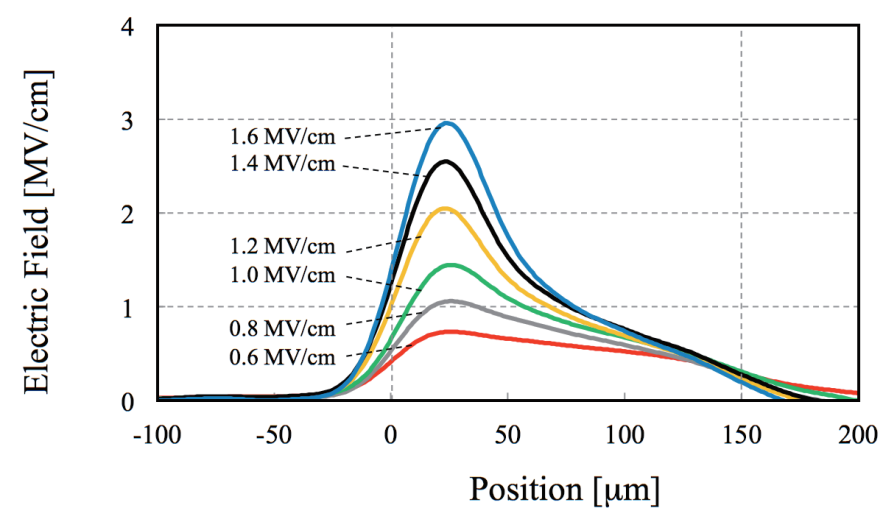

Fig. 7. (Color online) (a) Space charge distributions and (b) corresponding electric field distributions under various applied fields for additive-free LDPE with $\mathrm{SC}(+) / \mathrm{Al}(-)$. 


$$
E(x)=\frac{3}{2}\left(\frac{V}{d}\right)\left(\frac{d-x}{d}\right)^{\frac{1}{2}}
$$

This equation indicates that $E(x)$ is proportional to the square root of $d-x$. Note that the experimental profiles of $E(x)$ for additive-free LDPE with $\mathrm{SC}(+) / \mathrm{Al}(-)$ are entirely different from the theoretical profiles of $E(x)$ expressed by Eq. (3). Therefore, another conduction model is required to explain this experimental condition as the substitute of the SCLC model.

After the applied voltage is fixed for a few minutes in each step, the conduction current density $J$ is independent of the position $x$ because the space charge dynamics was scarcely observed at the latter stage in each step. Therefore, the relationship between the conductivity $\sigma(x)$ and the field strength $E(x)$ can be expressed as

$$
\sigma(x)=J / E(x)
$$

Now, we focus simply on the conductivity at the local thin area $(0<x<25 \mu \mathrm{m})$ for the additivefree LDPE with $\mathrm{SC}(+) / \mathrm{Al}(-)$. In this area, $E(x)$ is strongly enhanced by the high $F E F$, whereas $J$ does not increase with increasing $E$. From the experimental results, the conductivities at the local thin area corresponding to the field-concentrated area under the various applied fields can be calculated as $15 \times 10^{-14} \mathrm{~S} / \mathrm{m}$ for $E=0.6 \mathrm{MV} / \mathrm{cm}, 11 \times 10^{-14} \mathrm{~S} / \mathrm{m}$ for $E=0.8 \mathrm{MV} / \mathrm{cm}, 8.3 \times 10^{-14}$ $\mathrm{S} / \mathrm{m}$ for $E=1.0 \mathrm{MV} / \mathrm{cm}, 6.6 \times 10^{-14} \mathrm{~S} / \mathrm{m}$ for $E=1.2 \mathrm{MV} / \mathrm{cm}, 5.0 \times 10^{-14} \mathrm{~S} / \mathrm{m}$ for $E=1.4 \mathrm{MV} / \mathrm{cm}$, and $4.1 \times 10^{-14} \mathrm{~S} / \mathrm{m}$ for $E=1.6 \mathrm{MV} / \mathrm{cm}$. These calculation results indicate that the conductivity at the field-concentrated area decreases with increasing applied field. The physical reason for the decrease in the conductivity in the field-concentrated area is unclear at present. As far as we know, such complete current restriction under the very high field has not been observed in insulation materials. In the semiconductor research field, however, there are many reports on the restriction of the current through a very thin insulation layer used for small semiconductor devices. ${ }^{(19-21)}$ Several experimental formulas for the drift velocity and mobility of electrons and holes have been proposed. According to the experimental formulas proposed by Gummel ${ }^{(22)}$ and Thornber, ${ }^{(23)}$ the mobility under a very high field area tends to be reduced by the impurity scattering of electrons. The saturation of drift velocity due to the hot electron effect can be observed in very thin dielectrics whose thickness is comparable to the mean free path of electrons. Hot electrons are defined as electrons with kinetic energies substantially above thermal energies of a crystal lattice. Under a low applied field, the kinetic energies of electrons are much smaller than the thermal energies of the lattice. In such case, the mean free time of electrons, which corresponds to the time from a collision with the lattice to the subsequent collision, is independent of the field strength but depends on the thermal energies of the lattice. Since the electron mobility is proportional to the mean free time, it is also independent of the field strength as long as the applied field is low. Under a higher applied field, however, the mean free time of hot electrons decreases with increasing field strength because the hot electrons have a sufficient energy to produce scattering losses. Therefore, the electron mobility also decreases with increasing field strength. ${ }^{(24)}$

It should be remarked again that the current increases with the square of $E$ below $0.6 \mathrm{MV} / \mathrm{cm}$ but it saturates when the electrical stress is concentrated to the thin local area in front of the cathode. This phenomenon appears as if the anode comes close to the cathode to downscale the gap length. The drift velocity of carriers at the thin area subjected to the very high field of over a 
few $\mathrm{MV} / \mathrm{cm}$ may be restricted owing to the hot electron effect so that the measured current cannot be fitted onto the straight line corresponding to the SCLC model.

To further understand the current saturation phenomenon, it is important to observe the space charge distribution at the thin local area using a space charge measurement system with a much higher positional resolution. Unfortunately, the simultaneous measurement system that we made has a resolution of about $20 \mu \mathrm{m}$, which is too poor for observing the space charge dynamics in the very thin local area. Recently, a high-resolution space charge measurement system has been developed by Kumaoka et al. ${ }^{(25)}$ They reported that the positional resolution of this PEA system was enhanced up to a few $\mu \mathrm{m}$ by using a very thin piezoelectric sensor and by reducing the width of a voltage pulse to produce a very short acoustic wave from space charges. Such a high sensing technique will allow us to observe the space charge dynamics in the very thin area. We will also try to enhance the resolution of our PEA system and will measure the high field conduction and space charge dynamics for LDPE with various thicknesses on the order of $10^{-5} \mathrm{~m}$ as a future work.

\section{Conclusions}

Steady-state currents and space charge distributions were simultaneously measured for $150-\mu \mathrm{m}$-thick LDPE sheets subjected to stepwise increasing voltage. Three kinds of experimental conditions were arranged not only by adding the antioxidant agent but also by changing the electrode materials. Results are summarized as follows.

(1) Additive-free LDPE with $\mathrm{Al}(+) / \mathrm{Al}(-)$ electrodes

$J-E$ characteristics under a low applied field of below $0.6 \mathrm{MV} / \mathrm{cm}$ obeyed Ohm's law. By increasing the applied field stepwisely, both the space charge distribution and the electric field distribution were roughly in agreement with those based on the SCLC model, so that the steadystate current was proportional to $E^{2}$.

(2) Additive-containing LDPE with $\mathrm{SC}(+) / \mathrm{Al}(-)$ electrodes

The injection of positive charges from the SC electrode into the LDPE bulk was observed at a very low applied field. By increasing the applied field stepwisely, dense positive charges were injected in the LDPE bulk but they did not penetrate deeply into the bulk. This was because the antioxidant additive acted as deep trapping sites, so that the steady-state current was roughly proportional to $E^{2}$.

(3) Additive-free LDPE with $\mathrm{SC}(+) / \mathrm{Al}(-)$ electrodes

Under the low applied field of below $0.6 \mathrm{MV} / \mathrm{cm}$, both the $J-E$ characteristics and the space charge distributions were roughly in agreement with those based on the SCLC model. By increasing the applied field, however, the steady-state current saturated although a large number of positive space charges were accumulated in the LDPE bulk. We confirmed that the steadystate current was restricted when a large number of positive charges penetrated deeply into the bulk and finally they were frozen in front of the cathode, so that the field strength at the limited local area in front of the cathode surface was highly enhanced. These results indicated that the carrier mobility at the local thin area was reduced by the strong field concentration. 


\section{References}

1 A. Bradwell, R. Cooper, and B. Varlow: Proc. IEE 118 (1971) 247.

2 Y. Li and T. Takada: IEEE Electr. Insul. Mag. 10 (1994) 16.

3 N. Hozumi, T. Takeda, H. Suzuki, and T. Okamoto: IEEE Trans. Dielectr. Electr. Insul. 5 (1998) 82.

4 H. Kon, Y. Suzuoki, T. Mizutani, and N. Yoshifuji: IEEE Trans. Dielectr. Electr. Insul. 3 (1996) 380.

5 D. Fabiani, G. C. Montanari, and L. A. Dissado: IEEE Trans. Dielectr. Electr. Insul. 22 (2015) 45.

6 J. L. Auge, C. Laurent, D. Fabiani, and G. C. Montanari: IEEE Trans. Dielectr. Electr. Insul. 7 (2000) 797.

7 G. C. Montanari: IEEE Trans. Dielectr. Electr. Insul. 7 (2000) 309.

8 T. Takada, T. Mori, T. Kato, H. Miyake, and Y. Tanaka: IEEJ Trans. Fundam. Mater. 134 (2014) 258 (in Japanese).

9 T. Mori, H. Miyake, and Y. Tanaka: IEEJ Trans. Fundam. Mater. 133 (2013) 105.

10 M. Fukuma, M. Wadamori, M. Nagao, M. Kosakai, Y. Kohno, and T. Maeno: IEEJ Trans. Fundam. Mater. 121 (2001) 338 (in Japanese).

11 M. Fukuma, K. Fukunaga, and T. Maeno: J. Phys. D: Appl. Phys. 35 (2001) 14.

12 H. Yoshioka, K. Kadowaki, and I. Kitani: Elect. Eng. Jpn. 179 (2012) 10.

13 T. Kato, R. Onozawa, H. Miyake, Y. Tanaka, and T. Takada: IEEJ Trans. Fundam. Mater. 135 (2015) 75 (in Japanese).

14 G. Chen, T. Y. Tay, A. E. Davies, Y. Tanaka, and T. Takada: IEEE Trans. Dielectr. Electr. Insul. 8 (2001) 867.

15 M. A. Lampart: Phys. Rev. 103 (1956) 1648.

16 A. Many and G. Rakavy: Phys. Rev. 126 (1962) 1980.

17 K. Matsui, Y. Tanaka, T. Takada, T. Fukao, K. Fukunaga, T. Maeno, and J. M. Alison: IEEE Trans. Dielectr. Electr. Insul. 12 (2005) 406.

18 K. Matsui, Y. Tanaka, T. Takada, and T. Meno: IEEE Trans. Dielectr. Electr. Insul. 15 (2008) 841.

19 J. A. Cooper, Jr. and D. F. Nelson: J. Appl. Phys. 54 (1983) 1445.

20 S. A. Schwarz and S. E. Russek: IEEE Trans. Electron Devices ED-30 (1983) 1629.

21 M. Shirahata and C. Hamaguchi: Jpn. J. Appl. Phys. 25 (1986) 1040.

22 H. K. Gummel: IEEE Trans. Electron Devices ED-16 (1969) 64.

23 K. K. Thornber: J. Appl. Phys. 51 (1980) 2127.

24 S. M. Sze: Semiconductor Devices, Physics and Technology, 2nd ed. (Wiley, 2001) pp. 49, 75, and 275.

25 K. Kumaoka, H. Miyake, and Y. Tanaka: IEEJ Trans. Fundam. Mater. 136 (2016) 386 (in Japanese). 